\title{
Variation in hospital resource use and cost among surgical procedures using topical absorbable hemostats
}

This article was published in the following Dove Press journal:

ClinicoEconomics and Outcomes Research

6 November 2015

Number of times this article has been viewed

\author{
Derek Martyn' \\ Lisa M Meckley' \\ Gavin Miyasato' \\ Sangtaeck Lim² \\ Jerome B Riebman ${ }^{3}$ \\ Richard Kocharian ${ }^{3}$ \\ Jillian G Scaife' \\ Yajing Rao' \\ Mitra Corral ${ }^{2}$ \\ 'Trinity Partners, LLC, Waltham, \\ MA, USA; ${ }^{2}$ Global Health Economics \\ and Market Access, Ethicon, Inc., \\ Bridgewater, NJ, USA; ${ }^{3}$ Medical Affairs, \\ Ethicon, Inc., Bridgewater, NJ, USA
}

Correspondence: Mitra Corral

Ethicon, Inc., 737 US 22,

Bridgewater, NJ 08807, USA

Tel +19082182211

$\mathrm{Fax}+19082185435$

Email mcorral3@its.jnj.com
Background: Adjunctive hemostats are used to assist with the control of intraoperative bleeding. The most common types are flowables, gelatins, thrombins, and oxidized regenerated celluloses (ORCs). In the US, Surgicel ${ }^{\circledR}$ products are the only US Food and Drug Administration-approved ORCs.

Objective: To compare the outcomes of health care resource utilization (HRU) and costs associated with using ORCs compared to other adjunctive hemostats (OAHs are defined as flowables, gelatins, and topical thrombins) for surgical procedures in the US inpatient setting.

Patients and methods: A retrospective, US-based cohort study was conducted using hospital inpatient discharges from the 2011-2012 calendar years in the Premier Healthcare Database. Patients with either an ORC or an $\mathrm{OAH}$ who underwent a cardiovascular procedure (valve surgery and/or coronary artery bypass graft surgery), carotid endarterectomy, cholecystectomy, or hysterectomy were included. Propensity score matching was used to create comparable groups of ORC and OAH patients. Clinical, economic, and HRU outcomes were compared.

Results: The propensity score matching created balanced patient cohorts for cardiovascular procedure (22,718 patients), carotid endarterectomy (10,890 patients), cholecystectomy (6,090 patients), and hysterectomy (9,348 patients). In all procedures, hemostatic agent costs were $28 \%-56 \%$ lower for ORCs, and mean hemostat units per discharge were $16 \%-41 \%$ lower for ORCs compared to OAHs. Length of stay and total procedure costs for patients treated with ORCs were lower for carotid endarterectomy patients ( 0.3 days and US\$700) and for cholecystectomy patients ( 1 day and US\$3,350) (all $P<0.001$ ).

Conclusion: Costs and HRU for patients treated with ORCs were lower than or similar to patients treated with OAHs. Proper selection of the appropriate hemostatic agents has the potential to influence clinical outcomes and treatment costs.

Keywords: hemostatics, hemostatic techniques, blood transfusion, health care costs, surgical blood loss

\section{Introduction}

Tissue bleeding is a common risk for surgical procedures. Cardiac procedures have some of the highest risks of bleeding, with almost $50 \%$ of patients experiencing some type of bleeding-related consequence. ${ }^{1}$ Other common surgical procedures have bleeding rates ranging from $<10 \%$ to $35 \%{ }^{1}$ Uncontrolled bleeding is associated with increased risk of death, ${ }^{2}$ blood transfusions, and increased costs due to increased health care resource utilization (HRU). ${ }^{3}$

Adjunctive hemostats are intraoperative products that play a critical role in the control of bleeding in the operative setting, and were used in approximately $30 \%$ 
of procedures in $2010 .{ }^{4}$ There are a variety of adjunctive hemostatic agents that can reduce or stop bleeding. Four of the most common are: 1) oxidized regenerated celluloses (ORCs), 2) gelatins, 3) flowables, and 4) topical thrombins. ORCs and gelatins are mechanical hemostats. ${ }^{5}$ ORCs are a plant-derived product (cellulose), typically in a knitted or non-woven fabric form, which work by providing a surface for platelet adhesion and aggregation. ${ }^{6}$ Gelatins are porcineor bovine-derived products, which typically consist of a dry sponge. ${ }^{6,7}$ The gelatin products can be moistened with saline, ${ }^{6}$ in which case they would function like ORCs by providing a surface for platelet adhesion and aggregation. However, gelatins are also frequently moistened with thrombin. ${ }^{6}$ Flowables are also gelatin-based, but in the form of a flowable matrix ${ }^{7}$ that can be applied with a syringe and cannula. Thrombins are proteolytic enzymes that aid in the polymerization of the patient's own fibrinogen., Overall, there has been an increase over time in the use of adjunctive hemostatic agents to control bleeding in all surgical procedures. ${ }^{4}$

The objective of this study was to compare the HRU, costs (in US\$), and outcomes associated with using ORCs compared to other adjunctive hemostats (OAHs; flowables, gelatins, and topical thrombins) for surgical procedures performed in US inpatient settings in which both ORCs and OAHs were commonly utilized for hemostasis. The hemostatic effect of ORCs compared to other hemostat classes has been studied in a number of prospective clinical trials, ${ }^{8-12}$ and ORCs plus thrombin have been compared to other hemostat classes regarding their impact on inpatient length of stay (LOS) via a retrospective analysis of US hospital inpatient data. ${ }^{13}$ However, to the best of our knowledge, the current study is the first to use a cross-sectional dataset to retrospectively analyze the difference in a broad range of HRUs, costs, and outcome metrics for ORCs compared to other classes of adjunctive hemostats.

\section{Methodology \\ Data sources}

Data for this retrospective, observational cohort study were obtained from the Premier Healthcare Database (referred to as the Premier database). Premier is a consortium of US community and teaching hospitals that are non-profit and non-governmental, with approximately 6 million discharges annually. The Premier database covers approximately $25 \%$ of all hospital discharges in the US annually ${ }^{14}$ and is nationally representative. The database includes patient demographic and hospital characteristics, diagnoses, and procedures, as well as chargemaster data, which includes discharge-level resource utilization. This database contains a day of service-stamped log containing all billed items; laboratory, diagnostic, and therapeutic services rendered; and medications administered during the hospital stay. As an aggregated, de-identified, HIPAA compliant and statistically certified database, no institutional review board approval is required for analysis and publication. The Premier database was chosen for our current study due to its broad coverage of US inpatient procedure data allowing for generalizability to the entire US hospital market, and the ability to define the patient population and measure outcomes of interest.

\section{Study cohort}

The study cohort consisted of hospital discharges for adult patients (aged $\geq 18$ years) undergoing inpatient cardiovascular, carotid endarterectomy, cholecystectomy, or hysterectomy procedures during the 2011-2012 calendar years, in which adjunctive hemostats in the ORC, flowable, gelatin, or topical thrombin classes were utilized; Table 1 lists the products included in each class. The only ORC products currently approved for use in the US are Surgicel ${ }^{\circledR}$ (Ethicon, Inc., Somerville, NJ, USA) products; thus, the dataset only included Surgicel-brand ORCs. Flowables, gelatins, and thrombins were combined into a single comparison category, because these products are frequently used in combination

Table I Adjunctive hemostat products and classes

\begin{tabular}{|c|c|}
\hline Products and categories & Manufacturer \\
\hline \multicolumn{2}{|l|}{ ORCs } \\
\hline Surgicel ${ }^{\circledR}$ Original & Ethicon, Inc. (Somerville, NJ, USA) \\
\hline Surgicel Nu-Knit ${ }^{\circledR}$ & Ethicon, Inc. \\
\hline Surgicel SNoW'TM & Ethicon, Inc. \\
\hline Surgicel Fibrillar ${ }^{\circledR}$ & Ethicon, Inc. \\
\hline Surgicel ${ }^{\circledR}$, not otherwise specified & Ethicon, Inc. \\
\hline \multicolumn{2}{|l|}{ Flowables } \\
\hline Floseal $^{\circledR}$ & $\begin{array}{l}\text { Baxter Healthcare Corporation } \\
\text { (Hayward, CA, USA) }\end{array}$ \\
\hline Surgiflo ${ }^{\circledR}$ & Ethicon, Inc. \\
\hline Vitasure $^{\circledR}$ & $\begin{array}{l}\text { Stryker Corporation (Malvern, } \\
\text { PA, USA) }\end{array}$ \\
\hline Flowables, not otherwise specified & Unspecified \\
\hline \multicolumn{2}{|l|}{ Gelatin } \\
\hline Gelfoam $^{\circledR}$ & Pfizer, Inc. (New York, NY, USA) \\
\hline Surgifoam ${ }^{\circledR}$ & Ethicon, Inc. \\
\hline Gelatin powder & Multiple brands \\
\hline Gelatin, not otherwise specified & Unspecified \\
\hline \multicolumn{2}{|l|}{ Thrombin } \\
\hline Evithrom ${ }^{\circledR}$ & Ethicon, Inc. \\
\hline Recothrom $^{\circledR}$ & $\begin{array}{l}\text { ZymoGenetics, Inc. (Seattle, } \\
\text { WA, USA) }\end{array}$ \\
\hline Thrombi-Gel ${ }^{\circledR}$ & Pfizer, Inc. \\
\hline Thrombi-Pad ${ }^{\circledR}$ & Pfizer, Inc. \\
\hline Thrombin, not otherwise specified & Unspecified \\
\hline
\end{tabular}

Abbreviation: ORCs, oxidized regenerated celluloses. 
during a single procedure. Hemostat use was assessed on the basis of charge codes. Patients with missing data or who were treated with a combination of the ORCs and OAHs during the same procedure were excluded.

Cardiovascular procedures, carotid endarterectomy, cholecystectomy, and hysterectomy were selected for evaluation in the present study because both ORC and OAH usage were relatively common in these procedures, with each of these procedures including at least 3,000 discharges for $\mathrm{ORC}$ and $\mathrm{OAH}$ utilization. The original list of procedures considered for inclusion included arteriovenous graft, brain operations, coronary artery bypass graft surgery, carotid endarterectomy, cholecystectomy, and hysterectomy, operations on the kidney, prostatectomy, and valve surgery (Figure 1). Valve surgery and coronary artery bypass graft surgery were combined to create the cardiovascular procedure group since there was a significant overlap in the patients with these procedures. Procedures were identified based on the International Classification of Diseases, Ninth Revision, Clinical Modification (ICD-9-CM) procedures and current procedural terminology codes; Table S1 lists ICD-9 codes. Patients with multiple eligible discharges could be included in the cohort multiple times.

\section{Outcomes}

The primary endpoints for this study were LOS, adjunctive topical hemostatic product units per procedure, total procedure cost, transfusions during hospitalization, and mortality. LOS and total cost for each hospitalization were abstracted from the dataset as directly supplied by the hospital. The adjunctive topical hemostat product units per procedure abstraction was based on the count of the product units per discharge. Mortality was assessed using discharge status. Standard charge codes for transfused blood products and transfusion procedure codes were used to identify patient discharges with transfusions.

\section{Analysis}

Cohorts for each procedure were categorized according to the use of ORCs or OAHs. All analyses were performed separately for the indications of cardiovascular procedure, carotid endarterectomy, cholecystectomy, and hysterectomy. Descriptive statistics were calculated for demographic, clinical, and hospital characteristics. Outcome variables were compared between matched cohorts using standard statistical tests, eg, paired $t$-tests and McNemar's tests for continuous and categorical measures, relevant to the outcome variable data type and distribution.

To reduce the effect of treatment-selection bias, matched pairs of ORC and OAH patients were created using a propensity score matching (PSM) approach. ${ }^{15}$ PSM is frequently used in the medical literature as a means to adjust for confounding factors when using observational data to estimate the effect of treatments on outcomes. ${ }^{16}$ Once matched cohorts are created, several outcomes can be studied; this method provides an advantage over creating independent regression

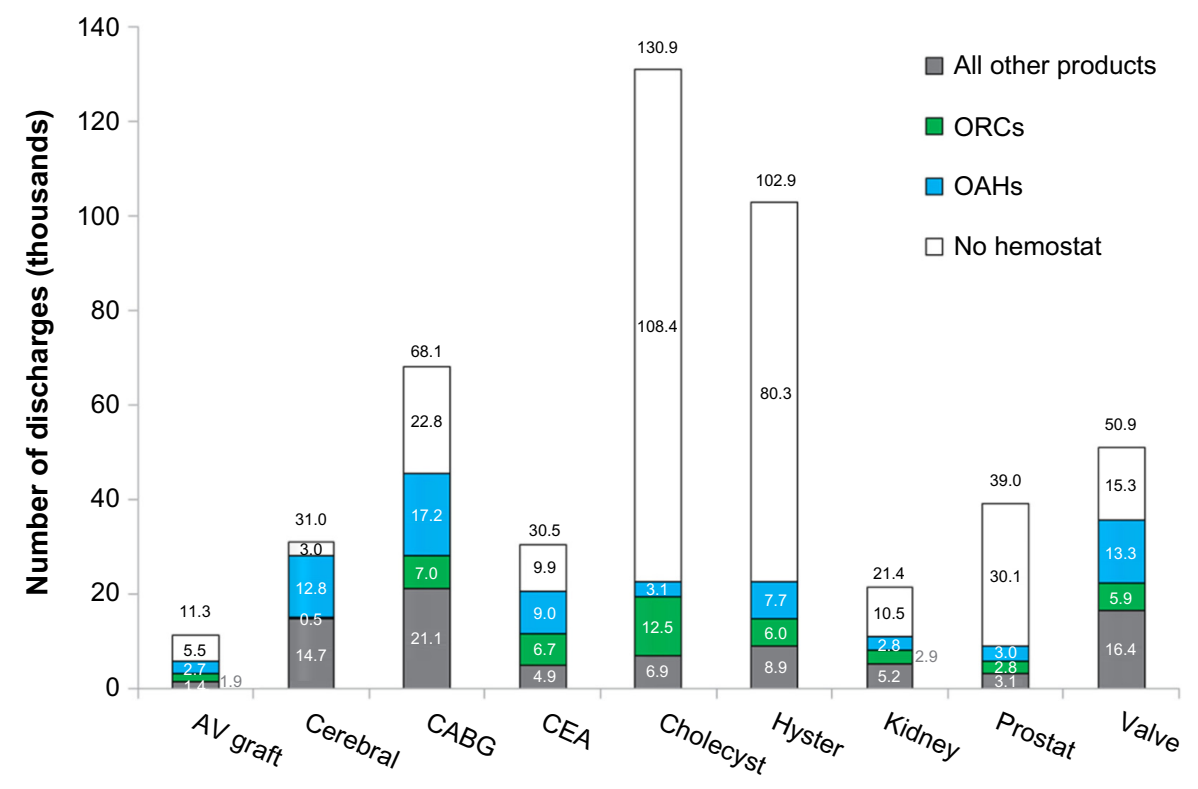

Figure I Distribution of adjunctive hemostat use by type of surgical procedure.

Abbreviations: ORCs, oxidized regenerated celluloses; OAHs, other adjunctive hemostats; CABG, coronary artery bypass grafting; CEA, carotid endarterectomy; Cholecyst, cholecystectomy; Hyster, hysterectomy; Prostat, prostatectomy; AV, arteriovenous. 
models for each outcome, because there is less concern about model misspecification. ${ }^{17}$ In this study, PSM was used to create matched patient cohorts based on their propensity to be treated with an ORC. To estimate a patient's propensity for a particular treatment, logistic regression models were fit, regressing on patient and hospital characteristics, specifically age, ethnicity, sex, and comorbidities; Table S2 identifies codes. Pre-existing bleeding conditions, also used to estimate propensity, are listed in Table S3. Teaching/non-teaching hospital status, hospital census region, and urban/rural hospital location were also considered in the estimation of treatment propensity. Additionally, to remove confounding factors due to the effects of other hemostat classes, concomitant use of non-ORC/non-OAH adjunctive hemostats were matched to isolate differences in outcomes between ORC and OAH classes. Patients were matched using a 1:1 greedy matching algorithm, ${ }^{18}$ and the balance of all patient and hospital characteristics was assessed. PSM was performed separately for each surgical procedure analyzed.

After the matched cohorts were created, differences between ORC and OAH cohorts were calculated for all outcome measures: LOS, total costs, transfusions, mortality, and ORC/OAH units per discharge for each procedure. Statistical differences between ORCs and OAHs for LOS were assessed with Wilcoxon signed-rank sum tests. Statistical differences for total procedure costs were calculated using Student's $t$-tests. Transfusion rates were evaluated based on $t$-tests for proportions. Fisher's exact tests were used to evaluate differences in mortality. Statistical analysis was performed using SAS version 9.2 software (SAS Institute Inc., Cary, NC, USA).

\section{Results}

\section{Cohort characteristics}

A total of 107,667 patients with a cardiovascular discharge during the 2-year study period were identified in the Premier hospital database. After accounting for the inclusion criteria, 11,813 patients treated with ORCs and 27,959 patients treated with OAHs were analyzed. For the carotid endarterectomy analysis, 30,480 patients were identified during the study period, with 6,689 ORC and 9,017 OAH patients meeting eligibility criteria. There were 130,931 cholecystectomy patients in the 2-year study period; 12,549 and 3,097 were treated with ORCs and OAHs, respectively. Finally, within the 102,882 identified hysterectomy patients, 5,982 were treated with ORCs compared to 7,742 with OAHs (Table 2).

The PSM produced cohorts of 22,718 patients for cardiovascular procedures (match rate of $96 \%$ relative to
ORC patients), 10,890 patients for carotid endarterectomy (match rate $81 \%$ relative to ORCs), 6,090 patients for cholecystectomy (match rate $98 \%$ relative to OAHs) and 9,348 patients for hysterectomy (match rate $78 \%$ relative to ORCs), with patients divided equally between ORCs and OAHs. After matching, patients in the ORC and OAH groups were similar with respect to demographic data, health status, hospital characteristics, and combinations with other hemostat types (Table 2).

\section{Topical adjunctive hemostat product units and costs}

Across all four procedure groups, the number of ORC product units used and cost per discharge were considerably lower than for OAH (Figure 2). Carotid endarterectomy gave the greatest differential, with $41 \%$ fewer units of ORCs used per discharge compared to OAHs $(P<0.001)$. Average total topical adjunctive hemostat cost was $47 \%$ less for ORCs than for OAHs, equating to a reduction of US\$88 per procedure. The other procedures also had considerably lower product units per procedure utilization for ORCs compared to OAHs (31\%, 18\%, and 16\% lower for cardiovascular procedures, cholecystectomy, and hysterectomy, respectively; $P<0.001$ for all three procedure types).

\section{Length of stay and total procedure costs}

Differences in mean LOS between the ORC and OAH cohorts were generally associated with total discharge costs. In carotid endarterectomy and cholecystectomy procedures, the shorter LOS for ORCs (Table $2 ; P<0.001$ for both procedure groups) aligned with the lower total procedure costs associated with the ORC cohorts in carotid endarterectomy ( $6 \%$ lower; $P<0.001)$ and cholecystectomy (14\% lower; $P<0.001)$. Conversely, in the cardiovascular group, a non-statistically significant 0.2-day longer LOS for procedures where ORCs were used $(P=0.948)$ was observed alongside a 3\% higher total procedure cost for ORCs $(P=0.003)$. Hysterectomy procedures displayed an interesting trend, whereby a longer LOS associated with ORCs $(P<0.001)$ was observed in conjunction with almost equivalent total procedure costs for the $\mathrm{ORC}$ and $\mathrm{OAH}$ cohorts $(P=0.542)$.

\section{Mortality and rate of transfusions during hospital stay}

Mortality rates for all procedures were low, $<4 \%$, and there was no statistically significant difference between ORCs and OAHs. A decrease in transfusion rates was found in the carotid endarterectomy and cholecystectomy ORC cohort 
Table 2 Baseline post-PSM cohort characteristics

\begin{tabular}{|c|c|c|c|c|c|c|c|c|c|c|c|c|c|c|c|c|}
\hline & \multicolumn{4}{|c|}{ Cardiovascular } & \multicolumn{4}{|c|}{ Carotid endarterectomy } & \multicolumn{4}{|c|}{ Cholecystectomy } & \multicolumn{4}{|c|}{ Hysterectomy } \\
\hline & \multicolumn{2}{|c|}{$\begin{array}{l}\text { ORC } \\
(n=11,359)\end{array}$} & \multicolumn{2}{|c|}{$\begin{array}{l}\text { OAH } \\
(n=11,359)\end{array}$} & \multicolumn{2}{|c|}{$\begin{array}{l}\text { ORC } \\
(n=5,445)\end{array}$} & \multicolumn{2}{|c|}{$\begin{array}{l}\text { OAH } \\
(n=5,445)\end{array}$} & \multicolumn{2}{|c|}{$\begin{array}{l}\text { ORC } \\
(n=3,045)\end{array}$} & \multicolumn{2}{|c|}{$\begin{array}{l}\text { OAH } \\
(n=3,045)\end{array}$} & \multicolumn{2}{|c|}{$\begin{array}{l}\text { ORC } \\
(n=4,674)\end{array}$} & \multicolumn{2}{|c|}{$\begin{array}{l}\text { OAH } \\
(n=4,674)\end{array}$} \\
\hline & $\mathbf{n}$ & $\%$ & n & $\%$ & $\mathbf{n}$ & $\%$ & n & $\%$ & $\mathbf{n}$ & $\%$ & n & $\%$ & $\mathbf{n}$ & $\%$ & n & $\%$ \\
\hline Age (mean) & 65.5 & - & 64.2 & - & 70.7 & - & 70.7 & - & 59.3 & - & 59.9 & - & 49.5 & - & 49.5 & - \\
\hline Male & 7,631 & $67 \%$ & 7,935 & $70 \% *$ & 3,157 & $58 \%$ & 3,110 & $57 \%$ & 1,506 & $49 \%$ & 1,489 & $49 \%$ & 0 & $0 \%$ & 0 & $0 \%$ \\
\hline \multicolumn{17}{|l|}{ Ethnicity } \\
\hline Caucasian & 8,636 & $76 \%$ & 8,760 & $77 \%$ & 4,593 & $84 \%$ & 4,604 & $85 \%$ & 2,151 & $71 \%$ & 2,158 & $71 \%$ & 3,079 & $66 \%$ & 3,046 & $65 \%$ \\
\hline African American & $\mathrm{I}, 35 \mathrm{I}$ & $12 \%$ & $\mathrm{I}, 294$ & $11 \%$ & 269 & $5 \%$ & 268 & $5 \%$ & 258 & $8 \%$ & 263 & $9 \%$ & 840 & $18 \%$ & 851 & $18 \%$ \\
\hline Hispanic & 43 & $<1 \%$ & 40 & $<1 \%$ & 18 & $<1 \%$ & 15 & $<1 \%$ & 49 & $2 \%$ & 45 & $1 \%$ & 68 & $1 \%$ & 79 & $2 \%$ \\
\hline Other & I,329 & $12 \%$ & 1,265 & $11 \%$ & 565 & $10 \%$ & 558 & $10 \%$ & 587 & $19 \%$ & 579 & $19 \%$ & 687 & $15 \%$ & 698 & $15 \%$ \\
\hline \multicolumn{17}{|l|}{ Comorbidity } \\
\hline Any malignancy & 539 & $5 \%$ & 523 & $5 \%$ & 248 & $5 \%$ & 232 & $4 \%$ & 347 & $11 \%$ & 375 & $12 \%$ & 987 & $21 \%$ & 938 & $20 \%$ \\
\hline Metastatic solid tumor & 14 & $<1 \%$ & 27 & $<1 \% *$ & 5 & $<1 \%$ & 3 & $<1 \%$ & 92 & $3 \%$ & 115 & $4 \%$ & 272 & $6 \%$ & 253 & $5 \%$ \\
\hline Diabetes & 4,610 & $41 \%$ & 4,705 & $41 \%$ & 1,896 & $35 \%$ & 1,939 & $36 \%$ & 810 & $27 \%$ & 809 & $27 \%$ & 518 & $11 \%$ & 527 & $11 \%$ \\
\hline Obesity & 2,317 & $20 \%$ & 2,345 & $21 \%$ & 553 & $10 \%$ & 527 & $10 \%$ & 650 & $21 \%$ & 649 & $21 \%$ & 843 & $18 \%$ & 839 & $18 \%$ \\
\hline Cerebrovascular disease & 1,228 & $11 \%$ & 1,163 & $10 \%$ & 5,434 & $100 \%$ & 5,433 & $100 \%$ & 76 & $2 \%$ & 95 & $3 \%$ & 29 & $1 \%$ & 35 & $1 \%$ \\
\hline $\begin{array}{l}\text { Peripheral vascular } \\
\text { disease }\end{array}$ & 4,275 & $38 \%$ & 4,133 & $36 \%$ & 1,290 & $24 \%$ & 1,365 & $25 \%$ & 233 & $8 \%$ & 205 & $7 \%$ & 27 & $1 \%$ & 22 & $0 \%$ \\
\hline Renal disease & 2,725 & $24 \%$ & 2,704 & $24 \%$ & 642 & $12 \%$ & 713 & $13 \% *$ & 602 & $20 \%$ & 617 & $20 \%$ & 139 & $3 \%$ & 127 & $3 \%$ \\
\hline Hypertension & 9,023 & $79 \%$ & 9,048 & $80 \%$ & 4,562 & $84 \%$ & 4,541 & $83 \%$ & 1,809 & $59 \%$ & $\mathrm{I}, 748$ & $57 \%$ & 1,545 & $33 \%$ & $\mathbf{1}, 555$ & $33 \%$ \\
\hline Congestive heart failure & 3,051 & $27 \%$ & 2,972 & $26 \%$ & 512 & $9 \%$ & 496 & $9 \%$ & 319 & $10 \%$ & 353 & $12 \%$ & 72 & $2 \%$ & 60 & $1 \%$ \\
\hline Myocardial infarction & 3,394 & $30 \%$ & 3,516 & $31 \%$ & 744 & $14 \%$ & 683 & $13 \%$ & 187 & $6 \%$ & 214 & $7 \%$ & 55 & $1 \%$ & 48 & $1 \%$ \\
\hline $\begin{array}{l}\text { Chronic pulmonary } \\
\text { disease }\end{array}$ & 3,453 & $30 \%$ & 3,248 & $29 \% *$ & 1,347 & $25 \%$ & 1,384 & $25 \%$ & 566 & $19 \%$ & 556 & $18 \%$ & 488 & $10 \%$ & 483 & $10 \%$ \\
\hline \multicolumn{17}{|c|}{ Pre-existing bleeding conditions } \\
\hline 0 & 7,203 & $63 \%$ & 7,147 & $63 \%$ & 4,508 & $83 \%$ & 4,474 & $82 \%$ & 2,100 & $69 \%$ & 2,099 & $69 \%$ & 3,816 & $82 \%$ & 3,861 & $83 \%$ \\
\hline I & 2,423 & $21 \%$ & 2,489 & $22 \%$ & 663 & $12 \%$ & 687 & $13 \%$ & 546 & $18 \%$ & 537 & $18 \%$ & 751 & $16 \%$ & 717 & $15 \%$ \\
\hline 2 & 1,117 & $10 \%$ & 1,076 & $9 \%$ & 199 & $4 \%$ & 197 & $4 \%$ & 250 & $8 \%$ & 241 & $8 \%$ & 78 & $2 \%$ & 74 & $2 \%$ \\
\hline $3+$ & 616 & $5 \%$ & 647 & $6 \%$ & 75 & $1 \%$ & 87 & $2 \%$ & 149 & $5 \%$ & 168 & $6 \%$ & 29 & $1 \%$ & 22 & $<1 \%$ \\
\hline \multicolumn{17}{|l|}{ Census region } \\
\hline Northeast & 2,335 & $21 \%$ & 2,013 & $18 \% *$ & 561 & $10 \%$ & 494 & $9 \% *$ & 433 & $14 \%$ & 405 & $13 \%$ & 824 & $18 \%$ & 844 & $18 \%$ \\
\hline Midwest & I,7I7 & $15 \%$ & $\mathrm{I}, 772$ & $16 \% *$ & 1,307 & $24 \%$ & 1,233 & $23 \% *$ & 769 & $25 \%$ & 727 & $24 \%$ & 845 & $18 \%$ & 860 & $18 \%$ \\
\hline South & 6,148 & $54 \%$ & 6,517 & $57 \% *$ & 3,044 & $56 \%$ & 3,110 & $57 \% *$ & 1,140 & $37 \%$ & 1,192 & $39 \%$ & 2,496 & $53 \%$ & 2,461 & $53 \%$ \\
\hline West & 1,159 & $10 \%$ & $\mathrm{I}, 057$ & $9 \% *$ & 533 & $10 \%$ & 608 & $11 \% *$ & 703 & $23 \%$ & 721 & $24 \%$ & 509 & $11 \%$ & 509 & $11 \%$ \\
\hline \multicolumn{17}{|l|}{ Bed size } \\
\hline$<200$ & 287 & $3 \%$ & 287 & $3 \%$ & 412 & $8 \%$ & 433 & $8 \%$ & 522 & $17 \%$ & 498 & $16 \% *$ & 583 & $12 \%$ & 583 & $12 \%$ \\
\hline $200-400$ & 4,657 & $41 \%$ & 4,657 & $41 \%$ & 2,431 & $45 \%$ & 2,388 & $44 \%$ & 1,046 & $34 \%$ & 1,176 & $39 \% *$ & 1,967 & $42 \%$ & 2,035 & $44 \%$ \\
\hline$>400$ & 6,415 & $56 \%$ & 6,415 & $56 \%$ & 2,602 & $48 \%$ & 2,624 & $48 \%$ & I,477 & $49 \%$ & $|, 37|$ & $45 \% *$ & 2,124 & $45 \%$ & 2,056 & $44 \%$ \\
\hline \multicolumn{17}{|l|}{ Location } \\
\hline Urban & 10,408 & $92 \%$ & 10,337 & $91 \%$ & 4,534 & $83 \%$ & 4,531 & $83 \%$ & 2,772 & $91 \%$ & 2,735 & $90 \%$ & 4,220 & $90 \%$ & 4,249 & $91 \%$ \\
\hline Rural & 951 & $8 \%$ & 1,022 & $9 \%$ & 911 & $17 \%$ & 914 & $17 \%$ & 273 & $9 \%$ & 310 & $10 \%$ & 454 & $10 \%$ & 425 & $9 \%$ \\
\hline \multicolumn{17}{|l|}{ Affiliation } \\
\hline Teaching & 5,902 & $52 \%$ & 5,902 & $52 \%$ & 2,084 & $38 \%$ & 2,167 & $40 \%$ & $\mathrm{I}, \mathrm{II} 4$ & $37 \%$ & 1,109 & $36 \%$ & 1,920 & $41 \%$ & 1,946 & $42 \%$ \\
\hline Non-teaching & 5,457 & $48 \%$ & 5,457 & $48 \%$ & 3,361 & $62 \%$ & 3,278 & $60 \%$ & $|, 93|$ & $63 \%$ & 1,936 & $64 \%$ & 2,754 & $59 \%$ & 2,728 & $58 \%$ \\
\hline
\end{tabular}

Notes: $* P$-value $<0.05$. Presence of statistically significant differences for sex, individual comorbidities, location, and affiliation were determined by $z$-test; differences for ethnicity, pre-existing bleeding conditions, census region, and bed size were determined by chi-square test.

Abbreviations: ORCs, oxidized regenerated celluloses; OAHs, other adjunctive hemostats; PSM, propensity score matching; n, number of patients.

compared to the OAH cohort ( $P<0.001$ for both procedures) (Table 3).

\section{Discussion}

We compared patients treated with Surgicel ORCs to patients treated with OAHs on cost and resource utilization metrics including LOS, units of hemostat product usage, and transfusions using hospital chargemaster data. After creating comparable cohorts of ORC and OAH patients with PSM, across the four procedures studied (cardiovascular procedure, carotid endarterectomy, cholecystectomy, and hysterectomy), adjunctive hemostatic agent costs were significantly lower when ORCs were used instead of OAHs. Other measures, such as transfusions and readmissions, were similar to or in favor of ORCs compared to OAHs for most procedure types. LOS and total procedure cost were lower for ORC patients 


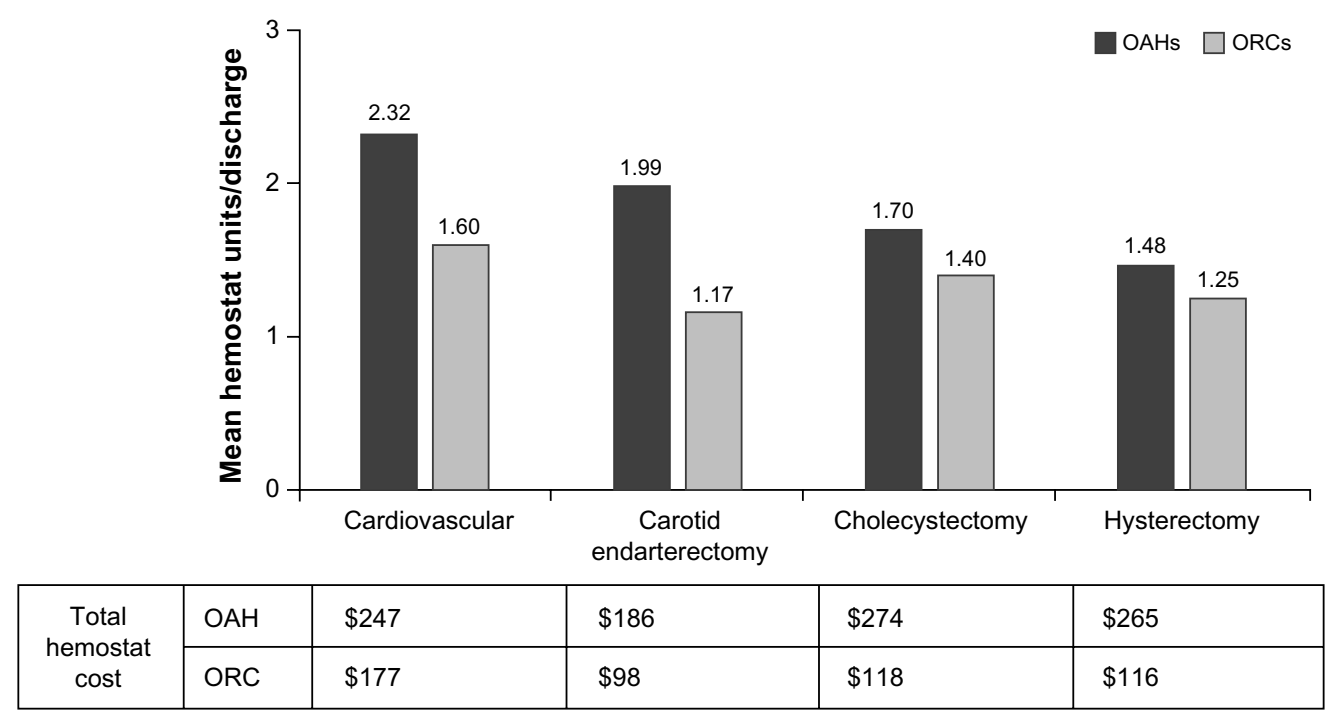

Figure 2 Hemostat units/discharge and total hemostat cost.

Notes: All differences between OAHs and ORCs for mean units/discharge total hemostat costs (US\$) were considered statistically significant at the $P<0.00 \mathrm{I}$ level. Costs for hemostatic agent classes included all hemostats used.

Abbreviations: ORCs, oxidized regenerated celluloses; OAHs, other adjunctive hemostats.

for both the carotid endarterectomy and cholecystectomy procedures. Overall, the average cost of ORCs was lower than OAHs, but the difference in overall procedure costs was greater than the cost difference of the hemostatic agents used.

Total procedure costs were linked to LOS. In this study, the total cost of treatment was lower for OAHs in the procedures in which LOS was lower. Cardiovascular disease was the only procedure in which the LOS and costs were not lower in ORC patients compared to OAH patients. It is not possible to discern from the current analysis why cardiovascular disease patients had higher costs when ORCs were used, but it may be due to different burdens of bleeding or different requirements for adjunctive hemostat characteristics during cardiovascular procedures, as compared to other procedures.

In the present study, cardiovascular patients' LOS ranged from 9.3 to 9.5 days; in a study conducted by Stokes et al, ${ }^{3}$ cardiovascular patients' LOS was similar, with an average of $\sim 8.5$ days. The Stokes study also found that bleedingrelated complications or blood product transfusions increased LOS in cardiac, vascular, and reproductive organ procedures by $4.8,9.3$, and 3.6 days, respectively, ${ }^{3}$ with cost increases of

Table 3 LOS, total procedure cost (US\$), transfusion rate, and 30-day readmissions: ORCs compared to OAHs

\begin{tabular}{|c|c|c|c|c|}
\hline & Cardiovascular & $\begin{array}{l}\text { Carotid } \\
\text { endarterectomy }\end{array}$ & Cholecystectomy & Hysterectomy \\
\hline \multicolumn{5}{|l|}{ LOS } \\
\hline $\mathrm{OAHs}$ & 9.3 & 3.0 & 8.1 & 3.1 \\
\hline ORCs & 9.5 & 2.7 & 7.1 & 3.4 \\
\hline$P$-value & 0.948 & $<0.00$ I & $<0.001$ & $<0.001$ \\
\hline \multicolumn{5}{|c|}{ Total procedure cost } \\
\hline $\mathrm{OAH}$ & $\$ 37,784$ & $\$ 11,282$ & $\$ 23,656$ & $\$ 11,033$ \\
\hline ORCs & $\$ 39,019$ & $\$ 10,580$ & $\$ 20,309$ & $\$ 10,898$ \\
\hline$P$-value & 0.003 & $<0.001$ & $<0.001$ & 0.542 \\
\hline \multicolumn{5}{|l|}{ Mortality } \\
\hline $\mathrm{OAHs}$ & $2 \%$ & $<1 \%$ & $3 \%$ & $<1 \%$ \\
\hline ORCs & $2 \%$ & $<1 \%$ & $2 \%$ & $<1 \%$ \\
\hline$P$-value & 0.467 & 0.654 & 0.137 & 0.297 \\
\hline \multicolumn{5}{|c|}{ Transfusion rate during hospital stay } \\
\hline $\mathrm{OAHs}$ & $43 \%$ & $5 \%$ & $19 \%$ & $14 \%$ \\
\hline ORCs & $44 \%$ & $4 \%$ & $16 \%$ & $13 \%$ \\
\hline$P$-value & 0.124 & $<0.00$ I & $<0.001$ & 0.303 \\
\hline
\end{tabular}

Abbreviations: ORCs, oxidized regenerated celluloses; OAHs, other adjunctive hemostats; LOS, length of stay. 
$\$ 10,000, \$ 15,000$, and $\$ 3,000$, respectively. More recently, Wright et al found that the frequency of adjunctive hemostatic agent use in major surgeries has increased rapidly over time. ${ }^{4}$ While studies have been conducted on the reduction of bleeding due to adjunctive hemostat use, or bleeding's impact on costs and resource utilization, there have been a limited number of studies on the impact of adjunct hemostats on both costs and outcomes.

A meta-analysis of studies focused on fibrin sealant use did not find a significant difference in the length of hospital stay compared to no adjunctive hemostat use. ${ }^{19}$ While there have been several studies on the effect of fibrin sealants, there has been very little published on the comparative effects and costs of flowables, thrombins, gelatins, or ORCs. A randomized controlled trial evaluating different hemostat options (gelatin, ORC, flowable, and thrombin) would be useful to confirm or refute the results of the present study. In a previous study of ORCs in cardiovascular, carotid endarterectomy, and brain/cerebral operations, the newer, advanced products, Surgicel SNoW ${ }^{\circledR}$ (Ethicon, Inc., Somerville, NJ, USA) and Surgicel Fibrillar ${ }^{\circledR}$ (Ethicon, Inc.), were associated with lower HRU and costs compared with Surgicel Original products. ${ }^{20}$ The present study found similar outcomes for all ORCs compared to OAHs. A future study designed to compare the newer "advanced" products to each other would further help to evaluate adjunctive hemostat options.

All observational, retrospective studies of secondary data have inherent limitations. Not all of the factors that influence a physician's choice of products to treat specific patients are represented in the current dataset. Additionally, because the patients were not randomized, we cannot be certain that there were not inherent differences between the specific procedures or patients receiving ORCs and OAHs that influenced patient outcomes. However, we attempted to account for this by using PSM to create similar groups for comparison. PSM cannot control for unmeasured characteristics, such as the use of medications prior to hospital admission not captured in a cross-sectional database such as Premier, which could influence hemostasis in the operative setting; PSM is also less powerful than randomization. However, the patient populations evaluated in the present study were made comparable for all observable characteristics, and were assumed to be similar on unmeasured characteristics such as pre-admission medication use.

There are also limitations to the chargemaster data within the Premier database. The data do not capture hospital readmissions to other facilities with different medical record systems and non-hospital-based office visits, and also do not include all outpatient data for all hospitals that submit inpatient data. Additionally, it is unclear whether treatment patterns and outcomes differ between hospitals that are within or outside of the Premier hospital database and whether these hospitals are representative. However, these results are most likely generalizable to many patients across the US, as approximately $25 \%$ of all hospital discharges are from a hospital within the Premier database. ${ }^{14}$ Results outside the US may differ because product availability varies by geography. Finally, as with all claims and chargemaster data, coding errors or omitted procedure/product codes could lead to misclassification of patients and potential bias in the results. However, since these data are used for payment, there is a strong incentive for accuracy.

Adjunctive hemostat use has the potential to reduce bleeding complications and associated resource use and costs associated with those complications. As adjunctive hemostat use rises, there is interest in understanding the relative benefits of different hemostat types with respect to clinical and economic outcomes. In the Premier database, over 370,000 cardiovascular, carotid endarterectomy, cholecystectomy, and hysterectomy procedures utilized adjunctive hemostats from 2011 to 2012. Based on this finding, one may estimate that in the US, for these procedures alone, approximately 750,000 adjunctive hemostat units are used annually in cardiovascular, carotid endarterectomy, hysterectomy, and cholecystectomy procedures.

\section{Conclusion}

In conclusion, while clinical outcomes were similar between procedures where ORCs and OAHs are used, ORC use was associated with fewer adjunctive hemostat units per procedure, as well as lower adjunctive hemostat spending when ORCs rather than OAHs were utilized. Additionally, in carotid endarterectomy, cholecystectomy, and hysterectomy procedures, ORC use was associated with lower overall LOS. In the present study, we also found that procedural adjunctive hemostat costs were at least $50 \%$ lower when ORCs were used compared to OAHs, with similar clinical outcomes. Utilizing more ORCs compared to OAHs during appropriate surgical procedures may have a meaningful impact on overall health care expenditures.

\section{Acknowledgments}

This study was funded by Ethicon, Inc. Materials in this manuscript were previously presented at the Society for the Advancement of Blood Management 2014 Annual Meeting, Houston, TX, September 11-13, 2014. Yogesh Soneji obtained funding and assisted with study design. 


\section{Disclosure}

JBR, SL, RK, and MC are all shareholders of Johnson \& Johnson and current or former employees of Ethicon, Inc. DM, LMM, GM, JGS and YR are all current or former consultants for Johnson \& Johnson and Bard, CR. The authors report no other conflicts of interest in this work.

\section{References}

1. Shander A. Financial and clinical outcomes associated with surgical bleeding complications. Surgery. 2007;142(4 Suppl):S20-S25.

2. Marietta M, Facchini L, Pedrazzi P, Busani S, Torelli G. Pathophysiology of bleeding in surgery. Transplant Proc. 2006;38(3):812-814.

3. Stokes ME, Ye X, Shah M, et al. Impact of bleeding-related complications and/or blood product transfusions on hospital costs in inpatient surgical patients. BMC Health Serv Res. 2011;11:135.

4. Wright JD, Ananth CV, Lewin SN, et al. Patterns of use of hemostatic agents in patients undergoing major surgery. $J$ Surg Res. 2014;186(1): 458-466.

5. Gabay M, Boucher BA. An essential primer for understanding the role of topical hemostats, surgical sealants, and adhesives for maintaining hemostasis. Pharmacotherapy. 2013;33(9):935-955.

6. Sundaram CP, Keenan AC. Evolution of hemostatic agents in surgical practice. Indian J Urol. 2010;26(3):374-378.

7. Fagan NL, Chau J, Malesker MA. Topical hemostats. US Pharmacist. 2010;35(11):HS2-HS8. Available from: http://www.uspharmacist.com/ content/d/feature/c/23860/. Accessed May 5, 2014.

8. Albala DM, Riebman JB, Kocharian R, et al. Hemostatis during urologic surgery: fibrin sealant compared with absorbable hemostat. Rev Urol. 2015;17:25-30

9. Fischer CP, Bochicchio G, Shen J, Patel B, Batiller J, Hart JC. A prospective, randomized, controlled trial of the efficacy and safety of fibrin pad as an adjunct to control soft tissue bleeding during abdominal, retroperitoneal, pelvic, and thoracic surgery. J Am Coll Surg. 2013;217:385-393.
10. Nativ O, Patel B, Shen J, Batiller J, Horn S, Hart JC. Safety and hemostatic efficacy of fibrin pad in partial nephrectomy: results of an open-label phase I and a randomized, standard-of-care-controlled phase I/II study. BMC Nephrol. 2012;13:147.

11. Nasso G, Piancone F, Bonifazi R, et al. Prospective, randomized clinical trial of the FloSeal matrix sealant in cardiac surgery. Ann Thorac Surg. 2009;88:1520-1526.

12. Sirlak M, Eryilmaz S, Yazicioglu L, et al. Comparative study of microfibrillar collagen hemostat (Colgel) and oxidized cellulose (Surgicel) in high transfusion-risk cardiac surgery. J Thorac Cardiovasc Surg. 2003; 126:666-670.

13. Krishnan S, Connor TM, Leslie R, Stemkowski S, Shander A. Choice of hemostatic agent and hospital length of stay in cardiovascular surgery. Semin Cardiothorac Vasc Anesth. 2009;13:225-230.

14. Premier, Inc. [webpage on the Internet]. Mission and vision. Charlotte, NC: Premier, Inc.; 2014. Available from: https://www.premierinc.com/ wps/portal/premierinc/public/aboutpremier/missionvision. Accessed May 5, 2014.

15. Rosenbaum PR, Rubin DB. The central role of the propensity score in observational studies for causal effects. Biometrika. 1983;70(1): $41-55$.

16. Austin PC. A critical appraisal of propensity-score matching in the medical literature between 1996 and 2003. Stat Med. 2008;27(12): 2037-2049.

17. Seeger JD, Williams PL, Walker AM. An application of propensity score matching using claims data. Pharmacoepidemiol Drug Saf. 2005; 14(7):465-476.

18. Steiner PM, Cook D. Propensity matching and propensity scores. In: Little TD, editor. The Oxford Handbook of Quantitative Methods in Psychology. Vol 1. New York: Oxford University Press; 2013.

19. Carless PA, Henry DA, Anthony DM. Fibrin sealant use for minimising peri-operative allogeneic blood transfusion [review]. Cochrane Database Syst Rev. 2003;2:CD004171.

20. Martyn D, Kocharian R, Lim S, et al. Reduction in hospital costs and resource consumption associated with the use of advanced topical hemostats during inpatient procedures. J Med Econ. 2015;18(6):1-8.
ClinicoEconomics and Outcomes Research

\section{Publish your work in this journal}

ClinicoEconomics \& Outcomes Research is an international, peerreviewed open-access journal focusing on Health Technology Assessment, Pharmacoeconomics and Outcomes Research in the areas of diagnosis, medical devices, and clinical, surgical and pharmacological intervention. The economic impact of health policy and health systems

\section{Dovepress}

organization also constitute important areas of coverage. The manuscript management system is completely online and includes a very quick and fair peer-review system, which is all easy to use. Visit http://www.dovepress.com/testimonials.php to read real quotes from published authors. 